\title{
A Strategy for Modeling Microstructure in Macroscopic Simulations of Welded Material
}

\author{
R.G. THIESSEN and I.M. RICHARDSON
}

With the increased precision in laser-welded structures, smaller and thinner parts can be joined. This magnifies the effect that microstructural changes due to the welding process have on the mechanical properties of the material. Although conventional welding processes are generally applied to work pieces of larger dimensions, the higher heat inputs and longer duration of the thermal cycle can have drastic effects on the microstructure. Multiscaled models can provide a method to achieve microscopic detail in a macroscopic simulation. The dual-mesh principle proposed here places microstructure domains at the nodes of a macroscopic finite-element (FE) calculation.

\section{INTRODUCTION AND MOTIVATION}

MICROSTRUCTURE in the heat-affected zone (HAZ) can have a significant impact on the mechanical properties of a weldment, including influences on strength, toughness, hardness, and crack resistance.$^{[1-3]}$ Both academia and industry have interest in this field since proper prediction and design can deepen current understanding of the joining process and lead to substantial savings in production costs and product efficiency. Unfortunately, the computational cost of calculating detail on the submicrometer scale for a work piece of millimeter scale or larger is very high. Simulations of this order thus demand an intelligent meshing scheme for greater detail in regions with large temperature and microstructure gradients and lesser detail where the gradients are not so large. With adaptive meshing techniques, the degree of detail can be varied temporally and spatially to provide optimum detail with minimum computational costs. Given the large variety of simulation techniques, it is generally accepted that certain methods are more suited to certain physical phenomena than others. Careful consideration can lead to an integration scheme that blends multiple methods in a single simulation. This could, for example, produce a method that combines the finite-element (FE) technique for macroscaled thermomechanical effects and a phase-field (PF) simulation to represent microstructure evolution. In addition, for simple geometry and constant welding conditions, quasisteady-state conditions can be assumed along the weld path, except for small weld-start and weld-end regions.

At this point, it should be emphasized that the coupled FE-PF approach presented in this work has the potential of providing much more information about microstructure morphologies than simpler phenomenological models. While semiempirical solutions, such as the Johnson-Mehl-Avrami-Kolomgrev (JMAK) based models, have been used for approximations of the phase fraction residing in a given volume in the material, there are limitations to these results. First, doubts have been raised as to the information provided from such phenomenological methods, whether they lend further understanding to

R.G. THIESSEN, Ph.D. Researcher, is with The Netherlands Institute for Metals Research (NIMR), 2628 AL, Delft, The Netherlands. I.M. RICHARDSON, Professor, is with Joining Technology, Technical University of Delft, 2628 AL, Delft, The Netherlands. Contact e-mail: r.g.thiessen@tnw.tudelft.nl

Manuscript submitted August 5, 2004. the physical processes of the transformation or whether they are simply an exercise in parameter fitting. Second, these relations were designed upon isothermal experimental results. Although some creative methods have been developed to apply the JMAK relations to piecewise isothermal steps ${ }^{[4]}$ the method is applicable only for constant heating rates. Finally, JMAK methods are inherently incapable of reflecting any geometrical effects in the microstructure, including grain orientation and shape. The proposed method could reflect these geometrical properties in the macrostructure by allowing the FE to take on the properties (such as anisotropy) of the microstructural domains that belong to the element.

With the proper modeling techniques, the simulation of welded material at the macroscopic and microstructural levels can be made tractable. This article describes some of the features of a strategy involving a combination of approaches.

\section{A DUAL-MESH STRATEGY}

The modeling of welded microstructure bridges many length and time scales, and requires more than intelligent meshing schemes to make simulations feasible. Refined meshes along the weld seam and an adaptive mesh ${ }^{[5]}$ around the moving heat source can resolve the heat flux and temperature distribution. However, for physically based models, where the evolution and morphology of the microstructure itself form the basis for the material mechanical properties, an undesirably large number of elements are required to produce the necessary level of detail. In this light, a dual-mesh strategy is proposed in which a fine, submesh resides at the nodal points of a coarse mesh. The fine submesh defines a small domain at which microstructure characteristics and evolution are calculated. These domains should be as small as possible to reduce computational costs; however, they must be large enough to contain the characteristic length of the entity to be described. In modeling the microstructure, this length would be of the same order of magnitude as the average grain diameter. Although the remainder of this work will apply the dual-mesh strategy only to the HAZ, the principles would also apply in the weld pool.

The dual-mesh strategy is designed to function as follows. The thermal field is calculated on a standard, three-dimensional FE mesh. Temperature and temperature gradient information at each node is then supplied to the submesh 


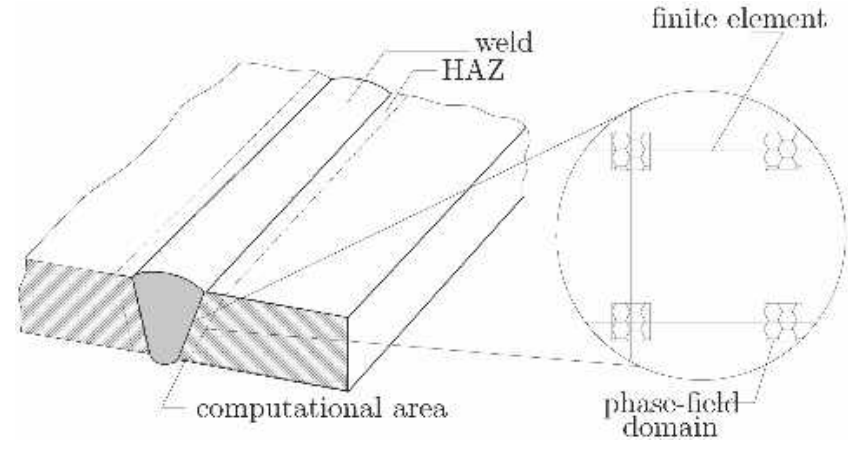

Fig. 1-Schematic representation of dual-mesh strategy in a macroscopic mesh. The zoomed-in area (right) illustrates a finite element with PF domains at each of the corner nodes.

residing at the node. Due to its strengths in capturing multiphase evolutions and geometrical properties of a microstructure, a multiphase $\mathrm{PF}^{[6]}$ is implemented at the submesh level. Since thermal diffusivity is several orders of magnitude higher than chemical diffusion, thermal gradients at the PF level can be linearized. The idea of a coarse thermal mesh to drive calculations of a finer mesh was also used in independent research by Ichikawa et al. ${ }^{[7]} \mathrm{A}$ salient difference with respect to the current strategy is that Ichikawa et al. meshed the entire body with a fine mesh, rather than with small, discrete submesh domains.

In order to elucidate the computational advantage of the dual-mesh strategy, a cross-sectional area near the weld pool is considered where the FE mesh has its smallest element size due to refinement and adaptive meshing. To resolve the edge of the weld pool for laser welding, for example, finite elements with an edge length of $200 \mathrm{~m}$ are used. Grain size could be on the order of 10 to $20 \mu \mathrm{m}$, which would require a PF domain size of $20 \times 20 \mu \mathrm{m}$.* Since one quarter of each

*The duration of the thermal cycle for conventional arc welding is somewhat larger, allowing grain growth to play a role. The larger weld pool could also be resolved with larger elements. These factors would change the requirements for the PF domain size.

$\mathrm{PF}$ domain lies in the finite element, a total PF domain area of $400 \mu \mathrm{m}^{2}$ inhabits each finite element. If the PF domain covered the entire finite element, the PF domain area would be 40,000 $\mu \mathrm{m}^{2}$. By considering discrete points, the dual-mesh strategy requires a densely meshed PF domain over only 1 pct of the area. If the entire simulation were able to fit into cached memory, this would translate into roughly 99 pct savings in computational time. However, due to the hierarchy of memory in modern computers, the increase in memory requirements for a given simulation can have an exponential effect on the total calculation time. ${ }^{[8]}$

Communication between the coarse mesh and the submesh domains is critical. The coarse FE mesh calculates the temperature distribution in time increments that are small enough to resolve the thermal history of points with the highest heating and cooling rates. For laser welding, this typically leads to time increments of no larger than $10^{-3}$ seconds. Thermal information is supplied to the submesh at each time-step. Even though chemical diffusion proceeds more slowly than thermal diffusion, the small grid-point spacing of the submesh can require smaller time-steps. The principle behind this is that the time-step should not be so large that the quantity in question (here, typically diffusing carbon or a proceeding phase front) could skip grid points within the time-step. This can also be stated as $\Delta t<$ $\Delta x / v$, and, with a submesh grid spacing of $10^{-7} \mathrm{~m}$, time-steps are commonly in the order of $t=10 \mathrm{~m} / \mathrm{s}$. The implied characteristic velocity on the order of $v=10 \mathrm{~m} / \mathrm{s}$ might seem high, but interface velocities of freshly nucleated grains with near infinite curvatures can easily approach these values. In the case of smaller time-steps for the PF domain, temperatures for the PF submesh are simply linearly interpolated for times between the time-temperature data supplied by the FE calculations.

When phase transformations cause distortion (such as with martensitic phase transformations) or cause a change in the thermomechanical properties (such as the discontinuous jump in the thermal expansion coefficient between ferrite and austenite), the flow of information is reversed and the submesh provides the FE mesh with updated material properties. The material properties for any given FE would be the average of the nodal values. These properties should be placed under a threshold criterion before updates occur in order to prevent unnecessary computational effort and avoid unnatural material property oscillations.

\section{BOUNDARY CONDITION AND MULTISCALE MODELING CONSIDERATIONS}

One of the most common boundary conditions used in welding models to reduce computational costs is symmetry. In the case of a weld bead following a straight path that bisects a regularly shaped body, assumptions of symmetry are valid and allow the computational problem to be halved. Another assumption, which holds for some conditions, is that of quasi-steady state. The conditions allowing this assumption start with a heat source moving with constant velocity. If any two points lying in a straight path parallel to the weld bead share the same thermal history, out of phase by the distance of separation divided by the weld velocity, then the region between the two points is said to be in quasi-steady state. In other words,

$$
T\left(x_{1}, t_{1}\right)=T\left(x_{2}, t_{2}+\frac{\Delta x}{v_{w}}\right), \text { with } x_{2}=x_{1}+\Delta x
$$

where $x$ is the distance separating the points on the path parallel to the weld and $v w$ is the velocity of the welding heat source. This is, of course, only valid sufficiently far from any edges that would lead to geometrical effects and produce inconsistencies. The power of the quasi-steady state refinement to the model is that, coupled with the assumption of homogeneity in the work piece, a four-dimensional problem (including the standard spatial coordinates $x y z$ and time) can be reduced to a representative two-dimensional cross section evolving with time. For a plate that is sufficiently thin, the two-dimensional cross section can be reduced to one dimension. The schematic representation of a welding simulation shown in Figure 2 depicts a weld advancing in the positive $y$-direction.** Possible

**Due to historical reasons, the FE code used dictates that the plane of symmetry is denoted by $x=0$.

paths for the analysis of quasi-steady state are denoted by the capital letters A through $\mathrm{G}$, and all paths lie parallel to the welding direction. 


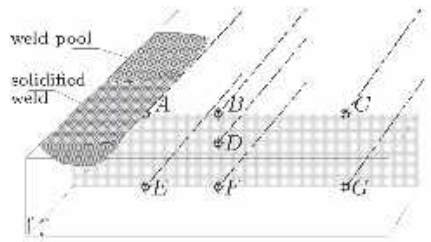

(a)

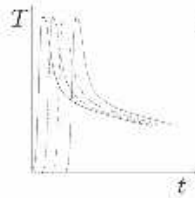

(b)

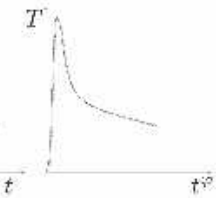

(c)
Fig. 2-(a) Cross-sectional view of a modeled plate. Possible paths of temperature calculations for evaluating quasi-steady state are indicated with the letters A through G. (b) Temperature histories for several nodes along one path. (c) Temperature histories transformed according to Equation [1].

To make a quantitative assessment of the thermal cycles, the time measurement must be adjusted by the appropriate phase shift via

$$
t^{\varphi}=t-\frac{\Delta x}{v_{w}}
$$

where $t$ is the in-phase time measurement for the thermal cycle. If comparing a series of points, $x$ is the distance of separation between the point in question and a set reference point. When the thermal cycles of the considered points are plotted with the in-phase time, variances in the curves can be evaluated. In the numerical setting with discretized time, an adequate representation of this variance could be based on the square of the differences divided by the average temperature according to

$$
\xi^{\prime}=\sum_{i=1}^{N} \frac{\sqrt{\left[T_{a}\left(x_{a}, t_{i}^{\varphi}\right)-T_{b}\left(x_{b}, t_{i}^{\varphi}\right)\right]^{2}}}{\frac{1}{2}\left[T_{a}\left(x_{a}, t_{i}^{\varphi}\right)+T_{b}\left(x_{b}, t_{i}^{\varphi}\right)\right]}
$$

Distances separating PF domains are dependent on the thermophysical properties of components and phases in the modeled alloy. Diffusion of components in the alloy play a large role in this criterion and will be dealt with first. Unlike most discretization methods, where refining the mesh leads to convergence toward a solution, placing PF domains too close to each other must be handled with care. In its simplest formulation, the dual-mesh strategy sets either periodic or symmetry boundary conditions on the PF domains. If the distance of separation between the PF domains is greater than a certain minimum, it is assumed that the boundaries of the PF domains are independent of each other. This assumption provides enormous computational savings, but it must be guaranteed that a component could not diffuse from one PF domain to its neighbor during the simulation, as shown in Figure 3. This would in effect lead to concentration fluxes that are not accounted for in the boundary conditions. For prolonged heat treatments, this condition could present challenges, but for the time frames considered in laser welding, the condition is usually easily satisfied. Conventional arc welding simulations produce thermal cycles whose duration is between that of the laser welding and standard heat treatments and would therefore require special care when setting up the models. Considering carbon as one of the fastest diffusing elements, and using the thermal cycle of a point approximately $50 \mu \mathrm{m}$ from the fusion line, the worst case scenario can be constructed. A diffusion coefficient of $D_{0}=1.26 \cdot 10^{-8} \mathrm{~cm}^{2} \cdot \mathrm{s}^{-1}$ and an activation energy of $Q=179.1 \mathrm{~kJ} \cdot \mathrm{mol}^{-1}$ for carbon

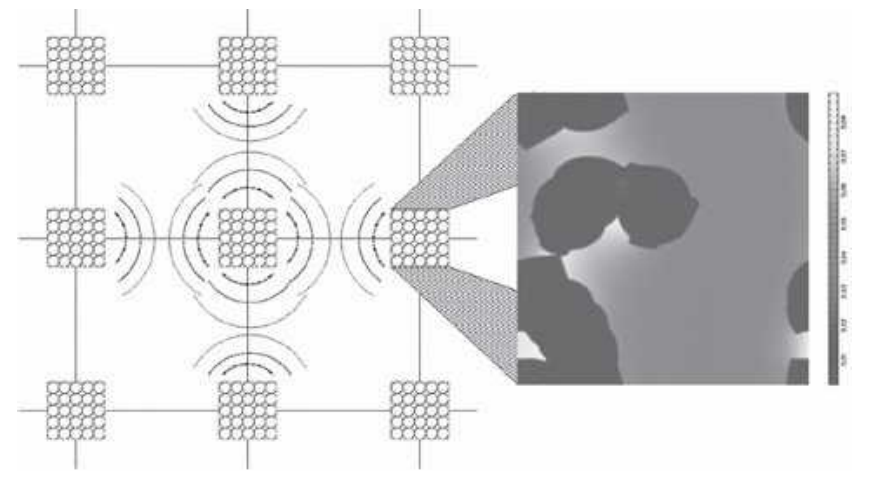

Fig. 3-On the left, a schematic depiction of PF domains residing at local grid points with surrounding regions that could be affected by diffusion. A zoomed-in view of a possible concentration field of a PF domain is shown on the right. The necessary separation distances are a function of the average velocity of the fastest diffusing element during a welding thermal cycle multiplied by the process time.

diffusion in $\delta$-ferrite* is used. To complete the worst case

*Average values from the literature for the diffusion coefficient of carbon in austenite are three orders of magnitude lower; thus, diffusion in a $\delta$-ferrite matrix provides the worst case scenario.

scenario, an inclusion of graphite will be considered as the source of carbon. Using a discretized form of the diffusion solution for the concentration at a point as a function of time, a nonisothermal case can be calculated. To evaluate the separations, it will be assumed that the threshold for deleterious effects to the boundary conditions is an unaccounted concentration change of $C=10 \mathrm{wt}$ pct. For the time scale of the welding process, only diffusion at temperatures above $773 \mathrm{~K}$ is considered nonnegligible. For a laser beam traveling speed of $v=40 \mathrm{~mm} \cdot \mathrm{s}^{-1}$, the time that a point next to the weld pool spends above $773 \mathrm{~K}$ is approximately 10 seconds. In this time, the concentration at a point $178 \mu \mathrm{m}$ from the graphite particle exceeds the threshold concentration. In other words, $178 \mu \mathrm{m}$ is the minimum distance of separation between PF domains. In contrast, autogenous arc welding** with an arc traveling speed

**A common example of autogenous arc welding uses a tungsten electrode and inert gas, leading to the acronym TIG.

of $v=3 \mathrm{~mm} \cdot \mathrm{s}^{-1}$ leads to the time spent above $773 \mathrm{~K}$ being approximately 20 seconds and the minimum distance of separation increasing to $1790 \mu \mathrm{m}$. A simulation of a heat treatment with an average temperature of $1173 \mathrm{~K}$ and a duration of 1800 seconds would require a PF domain separation of $1970 \mu \mathrm{m}$. For both the laser and TIG welding simulations, the minimum separation criterion could be relaxed with increasing distance from the fusion line. In all welding processes (and heat treatments), the minimal separation for a given material is basically a function of the peak temperature reached and the duration of near-peak temperatures. In Figure 4, the influence of laser beam traveling speed on the morphology of the thermal history can be seen. The sharper and lower peaks in temperature are the real reason behind the relation between welding speed and minimal distance of separation, as shown in Figure 5.

Considerations for the boundary conditions between PF domains also have mechanical aspects. Stresses generated 


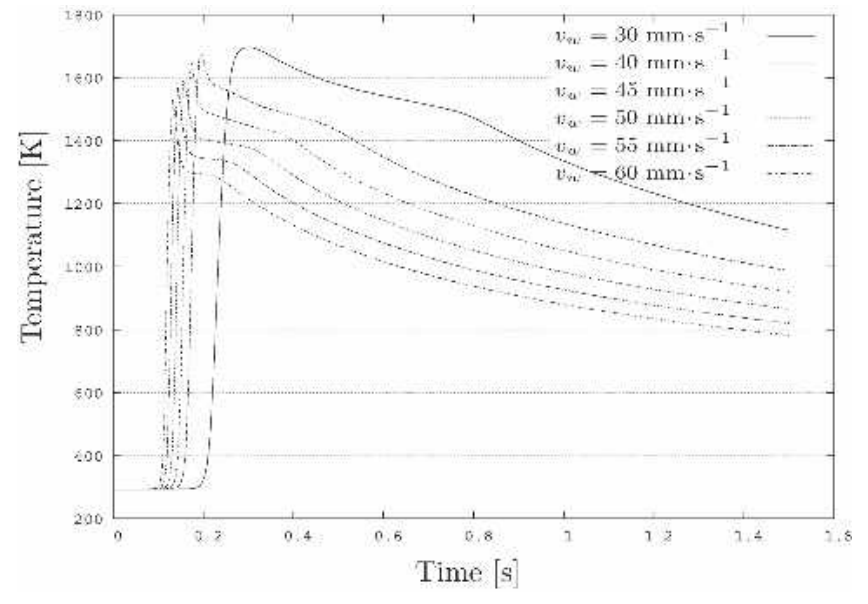

Fig. 4-The calculated temperature history for a point $50 \mathrm{~m}$ from the simulated fusion line of a laser weld. Note that due to varying weld pool sizes, these thermal histories are not from the same point. Lower welding speeds induce a higher peak temperature and a longer duration at near-peak temperatures.

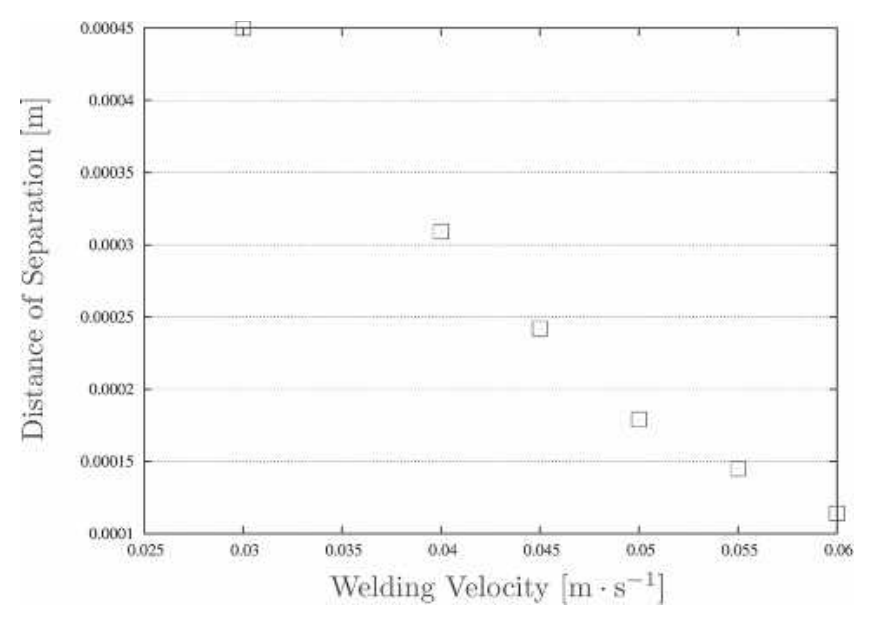

Fig. 5-The minimal distance of separation between PF domains based on the worst case scenario outlined in the text and the thermal histories shown in Figure 4.

from phase transformations must be transferable to the surrounding matrix. Considerable research has been carried out on the influence of stress fields on phase transformations, ${ }^{[9,10,11]}$ and this factor certainly must be accounted for in the presentation of the residual stresses from the welding process. The integration of displacive phase transformations in a matrix with significantly different mechanical properties has long been a field of research, with pioneering work done by Khachaturyan. ${ }^{[12]}$ At the microstructural level, the approach proposed by Khachaturyan can be applied where the transforming crystal is allowed to undergo transformation in a stress-free state. The surrounding matrix is then strained to accommodate the new shape of the transformed crystal. Since the martensite crystal has a significantly stiffer elastic modulus, the crystal can be reinserted in the matrix and the stress field from the matrix can be applied without appreciable deformation to the martensite crystal. This crude explanation of the Khachaturyan approach is a good thought experiment to imagine the stress field produced by a dis- placive phase transformation, but the implementation at the PF level has a simpler procedure. The transformations characteristic displacement is simply prescribed as a quasiDirichlet condition. The surrounding matrix is allowed to deform according to the standard elastoplastic material description. Mapping this material state to the macrolevel requires careful consideration. With a typically weaker matrix, displacements would be absorbed plastically within a PF domain. This means that displacements are not mapped to the FE mesh. The Neumann boundary conditions of the PF domain can take on standard symmetric or periodic properties, but the stress field must be mapped to the macroscopic scale. The details of this procedure are topics for continuing research. However, this does not impede the implementation of the dual-mesh strategy for diffusive, nondisplacive phase transformations.

\section{NUMERICAL SIMULATIONS}

For initial numerical simulations, the welding of AISI 316L with a high-powereddiode laser source was used. The material was chosen as an initial test case due to the extensive hightemperature mechanical and thermal property data that are available. ${ }^{[13]}$ Since the weld was performed in the conduction mode, a simple surface flux heat source was employed in a commercial finite element package. ${ }^{[5]}$ Thermal histories were evaluated based on qualitative and quantitative analysis of the experimental weld pool and the simulated temperature field. Once the thermal condition had reached quasi-steady state according to Eq. [3], a cross section was analyzed. Nodes that did not reach a maximum temperature of $773 \mathrm{~K}\left(500{ }^{\circ} \mathrm{C}\right)$ were considered as having negligible change in their microstructure and thus were not investigated with a PF submesh. AISI $316 \mathrm{~L}$ is considered an austenitic stainless steel and thereby avoids the displacive martensitic phase transformations. The formation and retention of $\delta$-ferrite were calculated. Due to the low heat conductivity of AISI 316L and the low heat input of the laser welding process, the HAZ for laser welding proved to be extremely thin. For a more rigorous test of the dual-mesh principle, some TIG welding simulations were also considered, with the intention of capturing the grain growth phenomenon. An FE model having undergone extensive experimental calibration $^{[13]}$ delivered the thermal field data for the PF domains in the TIG welding simulations.

The creation of a thermodynamically based material model for AISI $316 \mathrm{~L}$ called for the calculation of an equilibrium phase diagram. This was generated in the Thermo-Calc ${ }^{[14]}$ thermodynamic database, considering $\mathrm{Fe}, \mathrm{Cr}, \mathrm{Ni}, \mathrm{Mn}, \mathrm{Si}$, Mo, and $\mathrm{C}$. Due to paraequilibrium considerations, ${ }^{[15]}$ only $\mathrm{C}$ was treated as having appreciable diffusion during the time scale of the welding process. This led to the generation of a quasibinary phase diagram. Based on this diagram, the PF calculations could determine the driving forces for shrinkage, growth, or even nucleation of a given phase. The microstructure simulated was assumed to be completely austenitic in the initial state, founded on analysis of the base material.

Nucleation of $\delta$-ferrite is characterized by a given amount of overheating. Less overheating is required to nucleate at the triple junctions than at a two-grain interface. Interface interaction is described by the mobilities and activation energies, as studied by Krielaart et al. ${ }^{[16]}$ Figure 6 depicts the phase 


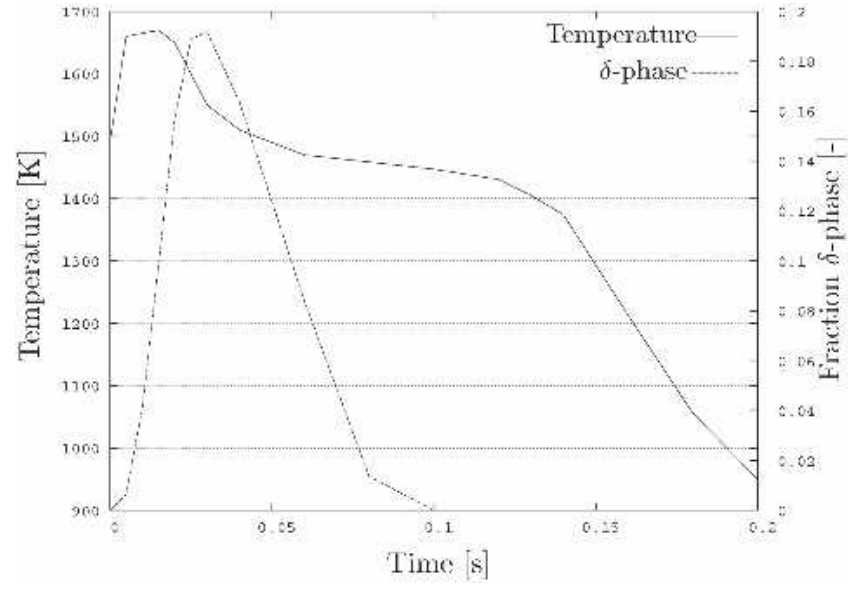

Fig. 6-Normalized thermal cycle (temperature divided by the maximum temperature, $1670 \mathrm{~K}$ ) plotted with the simulated phase fraction of $\delta$-ferrite in the HAZ of a laser weld. Note that the peak value of the $\delta$-ferrite fraction (0.38) occurs slightly after the peak temperature.

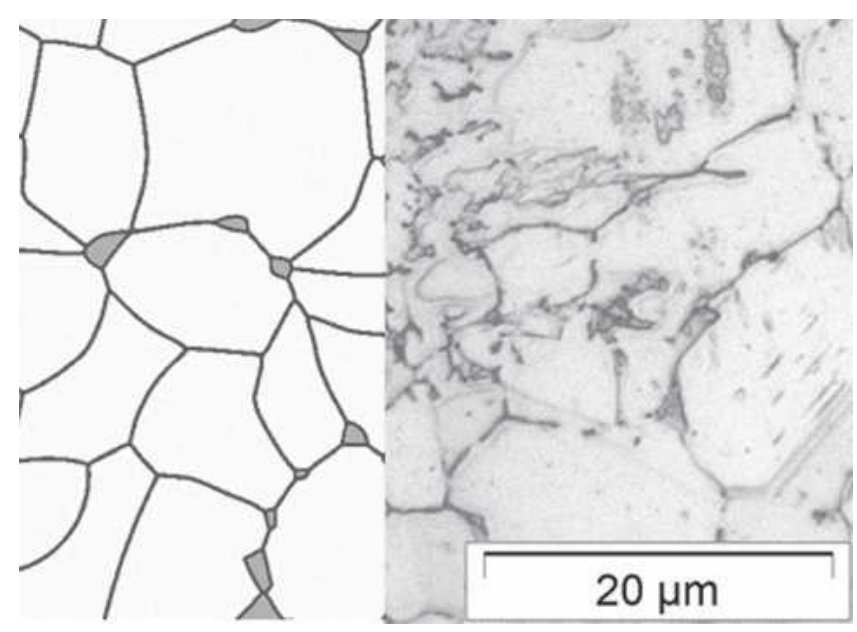

Fig. 7-Left: a simulated microstructure with $\delta$-ferrite (dark) in an austenitic matrix (light) in the HAZ of a laser weld. Right: a micrograph of $\delta$-ferrite (dark) in an austenitic matrix (light) in the HAZ of a laser weld.

fraction of $\delta$-ferrite with time. The phase fraction reaches a maximum of 38 pct slightly after the maximum temperature is reached. The calculated final microstructure is in good firstorder agreement with the actual microstructure in a similar position in the HAZ, shown in Figure 7.

Details of the parameters for nucleation and phase interaction will be treated in future publications. Of primary focus in this work are the principles of the dual-mesh strategy and coupling of thermal information from the macroscale to simulations at the microscale.

Recrystallization and grain growth calculations were compared with the experimental TIG weldments, as shown in Figure 8. Physical grain measurement was conducted along line segments parallel to the fusion line. This gave a statistically representative value for the average grain diameter at a given distance from the fusion line.

Grain boundary energy and interface mobility values for austenite were taken from the literature. ${ }^{[16,17,18]}$ Information

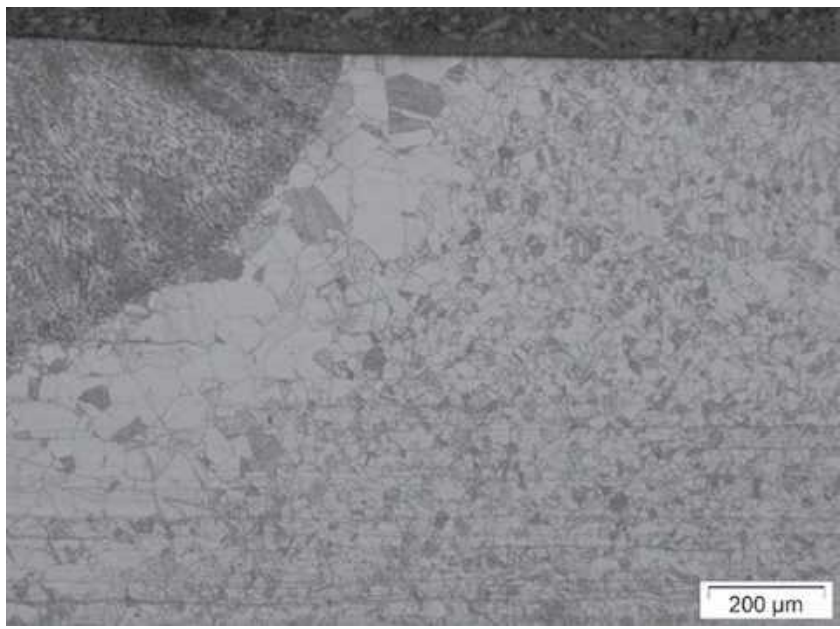

Fig. 8-Cross section of a weld made with a conventional TIG arc source. The edge of the weld pool can be seen in the upper left corner. A band of recrystallization and significant grain growth extends $300 \mathrm{~m}$ before blending via standard grain growth to the original grain size of the parent material.

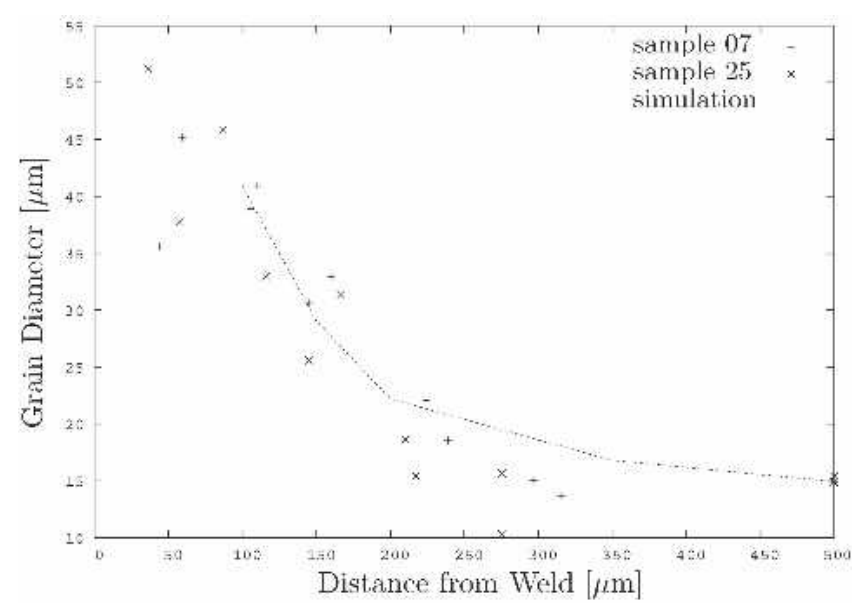

Fig. 9-Grain diameter $v s$ distance from fusion line in TIG welds. Simulated grain size values are joined with a dashed line. The pre-exponential coefficient and the activation energy for the interface submodel were taken as $0.45 \mathrm{~mol} \mathrm{~m}(\mathrm{~J} \mathrm{~s})^{-1}$ and $172.2 \mathrm{~kJ} \mathrm{~mol}^{-1}$, respectively.

over nucleation density of recrystallized grains was gained from annealed samples. The calculated grain size distribution is plotted with the measured values in Figure 9.

\section{VALIDATION AND DISCUSSION}

Most of the established microstructure models for welding treat only the cooling cycle. In agreement with other research, ${ }^{[19,20]}$ this initial investigation shows that the microstructure evolution during the heating cycle also deserves attention. Without simulation of the heating cycle, there is no physical basis for simulating the residual $\delta$-ferrite in an initially austenitic steel. Elmer et al. have made in-situ measurements of the phase transformations in steels ${ }^{[21]}$ with temporal resolution comparable to the simulations presented here. The measurements by Elmer et al. displayed the kinetic effects of the transformation in C-Mn steels and affirmed that 
the transformation was controlled by carbon diffusion through the austenite. The kinetic effect of the carbon diffusion is also reflected in the results of this work, with the maximum amount of $\delta$-ferrite occurring some time after the maximum temperature peak, as shown in Figure 6.

Recrystallization and grain growth in the HAZ of TIG weld simulations had good qualitative and quantitative agreement with experimental welds. The model treats interface mobility as a diffusive process with measured activation energies and is able to deliver a temperature-dependent mobility. The good agreement shown in Figure 9 confirms the sensitivity of the PF domains to both the temperature information from the macroscopic calculations and the temperature gradient data. Based on the model of interface mobility, an increase in temperature leads to an exponential increase in mobility. Due to the considerations in Section II, this requires a decrease in the time-step size. Using mobilities associated with temperatures under $1000 \mathrm{~K}$, the timestep size for the grain growth mesh was $>10$, but for temperatures above $1500 \mathrm{~K}$, the time-step size was limited to $<10$.

Computation time for the entire model was largely dependent on the number and size of PF domains. With the use of mesh refinement and adaptive meshing techniques, the finite element simulations of the thermal distribution were not a significant percentage of the total calculation time. Estimations of saved calculation time due to the use of the dual-mesh strategy are dependent on the size of the largest microscopic feature to be resolved in the PF domain. Models that were constructed to capture grain growth were therefore the most time consuming, since the domain size was required to be large enough to hold a representative amount of grains at their final size. The dependence of computation time on the PF domain size and time-step size was analyzed using a benchmark model. A nearly linear relationship exists between the area of the PF domain and the total computation time of the benchmark simulation, as seen in Figure 10. This dependence can be seen more strictly as a linear dependence on the number of grid points and the associated number of degrees of freedom within the PF domain. Simulations were kept small enough to avoid significant memory swapping, in which case computation times would increase drastically. This emphasizes the desire to keep PF domains as small as possible while still being large enough to capture the characteristic features of the microstructure.

Time-saving was also optimized by maximizing the timestep sizes for the various levels of modeling. The macroscopic thermal simulation could take advantage of the largest time-steps, with $\Delta t_{\mathrm{FE}} \sim 10^{-3} \mathrm{~s}$. The PF equations were solved on a somewhat smaller time-step size $\left(\Delta t_{\mathrm{PF}, \text { int }} \sim 10^{-4} \mathrm{~s}\right)$, using nested loops to call the concentration equations with an even smaller time-step $\left(\Delta t_{\mathrm{PF}, \text { conc }} \sim 10^{-6} \mathrm{~s}\right)$.

Regardless of the PF domain size, subroutines for nucleation and the numerical management required for additional phases had significant impact on the computation time. Figure 11 shows calculation time per simulated time-step. Nucleation of the $\delta$-ferrite occurred at $t \sim 0.01 \mathrm{~s}$ and ceased by $t \sim$ $0.03 \mathrm{~s}$. As the prescribed temperature dropped, $\delta$-ferrite grains shrank. The smallest $\delta$-ferrite grains vanished at $t \sim 0.07 \mathrm{~s}$, leading to the drop in calculation time. With falling temperature, interface mobility also decreased, leading to a stagnant

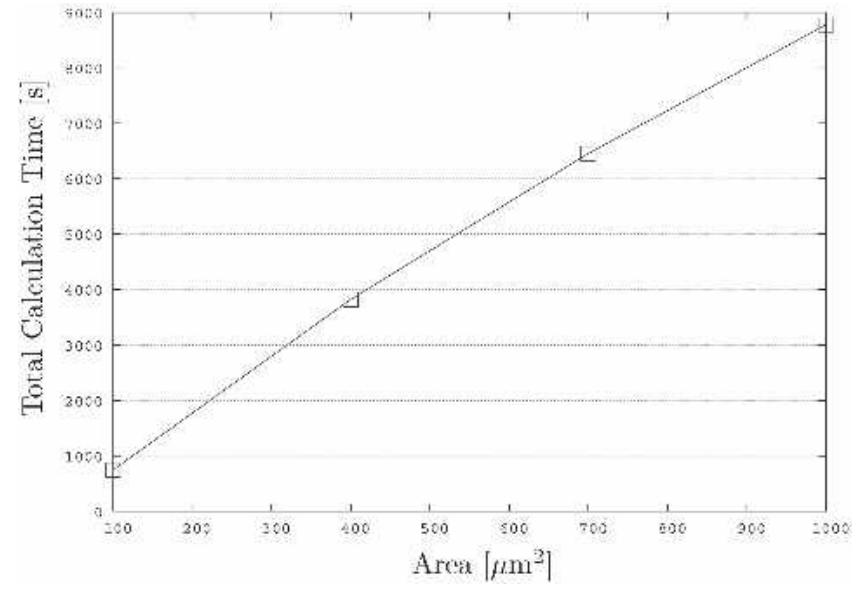

Fig. 10 - Total calculation time as a function of PF domain area. An approximately linear relationship is displayed. The slight deviation toward shorter times for smaller PF domains is likely a result of some processes being carried out entirely in cached memory.

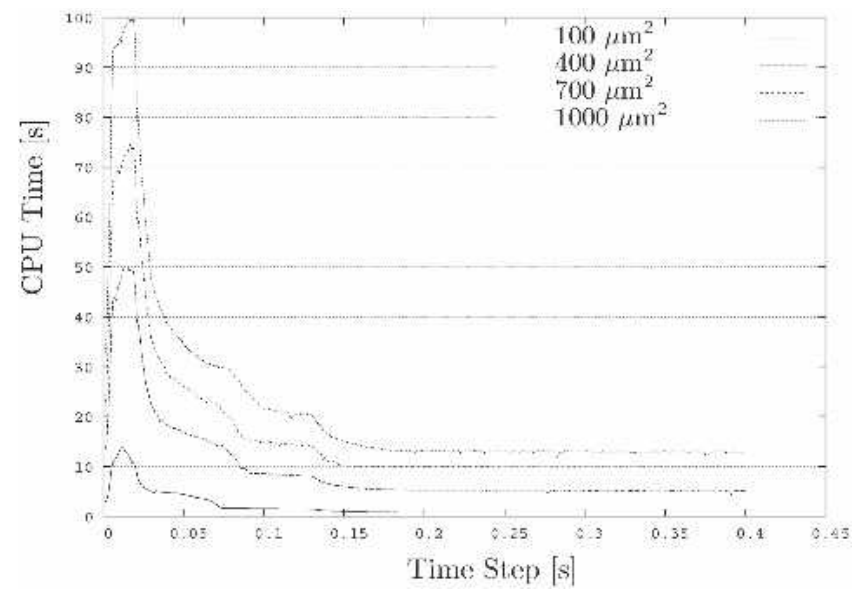

Fig. 11-Calculation time per time-step for increasing PF domain size. The noticeable peak in calculation time is due to nucleation of $\delta$-ferrite and the accompanying interaction with the parent phase. Computation time comes to a plateau once the temperature-dependent interface mobility practically freezes the grain boundaries.

microstructure by $t \sim 0.17 \mathrm{~s}$, with the accompanying plateau in the calculation time.

\section{CONCLUSIONS AND OUTLOOK}

The important result shown here is that the PF domain can accept a macroscopically calculated thermal field to drive a microscopic simulation that agrees well with experimental results while avoiding nonphysical fitting parameters. In essence, the dual-mesh strategy assumes that the PF domains are miniature view ports into a continuous domain. They are therefore part of the continuous domain, but separated sufficiently from each other to avoid overlapping of chemical influences on each other due to diffusion. The separation on the microscopic scale is appreciable, but on the meso- to macroscopic scale, the PF domains are close enough to each other to provide a semicontinuous representation of the microstructure. The dual-mesh strategy provides a method for computationally tractable microstructure simulations of macroscopic 
work pieces. These simulations also provide new insight into the evolution of microstructure in the HAZ and a link from the microstructure to the macroscopic mechanical properties of welded material.

\section{REFERENCES}

1. T.A. Palmer, J.W. Elmer, and J. Wong: Sci. Technol. Welding Joining, 2002, vol. 7 (3), pp. 159-71.

2. S.S. Babu, S. David, J.M. Vitek, and R.W. Reed: Sci. Technol. Welding Joining, 2001, vol. 6, pp. 31-40.

3. W. Zhang, J.W. Elmer, and T. DebRoy: Scripta Mater., 2002, vol. 46 (10), pp. 753-57.

4. P. Kruger: J. Phys. Chem. Solids, 1993, vol. 54 (11), pp. 1549-55.

5. MSC.Marc 2003 Manual Technical Report, MSC.Software Corporation, 2003.

6. I. Steinbach, F. Pezzolla, B. Nestler, M. See $\beta$ elberg, R. Prieler, G.J. Schmitz, and J.L.L. Rezende: Physica D, 1996, vol. 94 (3), pp. 135-47.

7. K. Ichikawa, A. Nagomi, T. Koseki, and Y. Fukuda: Numerical Analysis of Weldability, Institute of Materials, Graz, 2001.

8. I. Steinbach and M. Apel: private communications, Access e.V., Aachen Germany, 2004.

9. M.A. Fradkin: Phys. Rev. B, 1994, vol. 50 (22), pp. 16326-39.
10. A. Artemev, Y. Wang, and A.G. Khachaturyan: Acta Mater., 2000, vol. 48 (10), pp. 2503-18.

11. A. Artemev and A.G. Khachaturyan: Shape Memory Mater., 2000, vol. 327 (3), pp. 347-50.

12. A.G. Khachaturyan: Theory of Structural Transformations in Solids, John Wiley \& Sons, New York, NY, 1983.

13. E. Aa vd: Master's Thesis, Technical University of Delft, Delft, The Netherlands, 2002.

14. B. Sundman, B. Jansson, and J. Andersson: "The Thermo-Calc Databank System Technical Report", CALPHAD, Stockholm, Sweden, 1985.

15. J. Odqvist, B. Sandman, and J. Agren: Acta Mater., 2003, vol. 51, pp. 1035-43.

16. G.P. Krielaart and S. van der Zwaag: Mater. Sci. Eng. A-Struct. Mater Prop. Microstr. Processing, 1998, vol. 246 (1-2), pp. 104-16.

17. S.I. Vooijs, Y. Van Leeuwen, J. Sietsma, and S. Van der Zwaag: Metall. Mater. Trans. A-Phys. Metall. Mater. Sci., 2000, vol. 31 (2), pp. 379-85.

18. L. Murr: Interfacial Phenomena in Metals and Alloys, Addison-Wesley, Reading, MA, 1975.

19. G.Y. Zhang, T. Jing, and B.C. Liu: Int. J. Cast Met. Res., 2002, vol. 15 (3), pp. 237-40.

20. S.S. Babu, J.W. Elmer, J.M. Vitek, and S.A. David: Acta Mater., 2002, vol. 50 (19), pp. 4763-81.

21. J.W. Elmer, T.A. Palmer, W. Zhang, B. Wood, and T. DebRoy: Acta Mater., 2003, vol. 51 (12), pp. 3333-49. 